\title{
The deletion of bacterial dynamin and flotillin genes results in pleiotrophic effects on cell division, cell growth and in cell shape maintenance
}

Felix Dempwolff ${ }^{1,2 \dagger}$, Hanna M Wischhusen ${ }^{1,3+}$, Mara Specht ${ }^{1}$ and Peter L Graumann ${ }^{1,2^{*}}$

\begin{abstract}
Background: In eukaryotic cells, dynamin and flotillin are involved in processes such as endocytosis and lipid raft formation, respectively. Dynamin is a GTPase that exerts motor-like activity during the pinching off of vesicles, while flotillins are coiled coil rich membrane proteins with no known enzymatic activity. Bacteria also possess orthologs of both classes of proteins, but their function has been unclear.

Results: We show that deletion of the single dynA or floT genes lead to no phenotype or a mild defect in septum formation in the case of the dynA gene, while dynA floT double mutant cells were highly elongated and irregularly shaped, although the MreB cytoskeleton appeared to be normal. DynA colocalizes with FtsZ, and the dynA deletion strain shows aberrant FtsZ rings in a subpopulation of cells. The mild division defect of the dynA deletion is exacerbated by an additional deletion in ezrA, which affects FtsZ ring formation, and also by the deletion of a late division gene (div/B), indicating that DynA affects several steps in cell division. DynA and mreB deletions generated a synthetic defect in cell shape maintenance, showing that MreB and DynA play non-epistatic functions in cell shape maintenance. TIRF microscopy revealed that FloT forms many dynamic membrane assemblies that frequently colocalize with the division septum. The deletion of dynA did not change the pattern of localization of FloT, and vice versa, showing that the two proteins play non redundant roles in a variety of cellular processes. Expression of dynamin or flotillin T in eukaryotic S2 cells revealed that both proteins assemble at the cell membrane. While FloT formed patch structures, DynA built up tubulated structures extending away from the cells.
\end{abstract}

Conclusions: Bacillus subtilis dynamin ortholog DynA plays a role during cell division and in cell shape maintenance. It shows a genetic link with flotillin $\mathrm{T}$, with both proteins playing non-redundant functions at the cell membrane, where they assemble even in the absence of any bacterial cofactor.

\section{Background}

The dynamin protein superfamily is a large group of mechanochemical GTPases. Members of this family play an important role in vesicle formation, clathrin-dependent endocytosis, renewal of membrane components, and the division of organelles [1,2]. Dynamin-like proteins have a characteristic arrangement of an N-terminal GTPase

\footnotetext{
* Correspondence: peter.graumann@biologie.uni-freiburg.de

${ }^{\dagger}$ Equal contributors

'Mikrobiologie, Fachbereich für Biologie, University of Freiburg,

Schänzlestraße 1, Freiburg 79104, Germany

${ }^{2}$ LOEWE Center for Synthetic Microbiology, SYNMIKRO, Philipps-University

Marburg, Marburg 35043, Germany

Full list of author information is available at the end of the article
}

domain, a central domain and a GTPase effector domain [3]. Canonical dynamin has two additional domains, a pleckstrin homology domain and a C-terminal proline and arginine-rich domain that mediates interaction with proteins and lipids [4]. The GTPase domain couples GTP hydrolysis with a mechanical reaction that can confer motor-like functions. The middle domain is only poorly conserved and functions in multimerization of dynaminlike proteins. The effector domain serves in stimulation of GTPase activity and in the interaction of dynamin molecules. It contains characteristic heptad repeat regions that can form coiled coils, and which are relevant for dynamin interactions $[3,5]$. In spite of their similar general

\section{Biomed Central}


arrangement, dynamin-like proteins are highly divergent in their individual setup, probably reflecting the broad spectrum of cellular functions they are involved in $[4,6]$.

The GTPase motifs within the GTPase domain show similarity to regulatory Ras-like GTPases [7], however, the domain is much larger than that of regulatory GTPases, and does not require additional stimulatory proteins, but instead is 100 fold enhanced through oligomerization. The domain displays low GTP affinity (10 to $100 \mu \mathrm{M})$, but high GTPase activity. Purified dynamin has been shown to self-organize into rings and helical structures that are able to attach to lipid membranes and to distort them into large tubular structures. Addition of GTP gives rise to a conformational change and to a constriction, which ultimately leads to a fragmentation of the membrane. Some dynamin-like proteins have a high affinity to negatively charged phospholipids $[3,4,6]$, indicating that membrane composition and lipid rafts may be important for the localization of dynamins.

One of the best understood tasks performed by dynamin is pinching off of clathrin-coated vesicles. Dynamin assembles like a collar around clathrin-coated membrane invaginations and through GTP hydrolysis driven conformational change dissects the vesicle from the membrane $[8,9]$. In addition to this mechanical role, dynamin is discussed to be responsible for recruiting additional factors to the clathrin pits to facilitate and regulate the formation of the vesicles [10].

Interestingly, many bacterial genomes also contain potential dynamin-like proteins. The crystal structure of the protein termed BDLP (bacterial dynamin-like protein) from the filamentous cyanobacterium Nostoc punctiforme revealed that indeed, this protein has a typical dynamin GTPase domain, a neck domain, and an end domain [11]. Structural analysis of BDLP suggests that it operates as a homodimer as smallest unit. The purified protein shares several properties with dynamins: it self-assembles into tubular structures containing radial spokes, which tubulate membranes [12]. In vivo, BLDP localizes as irregular focuslike assemblies at the cell membrane [11]. Bacillus subtilis is a model organism for Gram positive bacteria and contains a predicted dynamin-like protein, DynA. DynA has recently been shown to be able to bind to membranes and to mediate membrane fusion in vitro, even in the absence of GTP [13]. However, there is no information yet on the function of bacterial dynamin-like proteins in vivo. A possible function in cell division has been proposed [13]. FtsZ is a tubulin ortholog that initiates cytokinesis by forming a ring structure at the cell centre. FtsZ recruits further proteins that eventually lead to the formation of a septum between the separated sister chromosomes $[14,15]$. In E. coli, proteins are assembled in a rather linear pathway [16], while in B. subtilis, a time delay exists between early recruited proteins (such as FtsA and ZapA) and late division proteins (such as FtsL and DivIb), indicating that proteins are recruited as complexes rather than singly [17]. Late division proteins include penicillin-binding proteins (Pbps) that synthesize the cell wall between the daughter cells. For growth as rods, actin-like MreB proteins are essential in many bacteria, interacting with Pbps and other membrane proteins involved in cell wall synthesis $[18,19]$. According to one theory, MreB forms filamentous structures underneath the cell membrane that direct the incorporation of new cell wall material via an interaction with the synthetic enzymes. The depletion of MreB leads to the generation of round cells that eventually lyse [20], showing that the protein plays an important function in cell shape maintenance.

Eukaryotic and prokaryotic membranes contain an asymmetric distribution of lipids. Especially cholesterol and sphingolipids in eukaroytes cluster into so called lipid rafts [21]. These dynamic microdomains also cluster proteins, many of which are involved in the transport of membrane components and in signal transduction. Flotillins are a class of membrane proteins that are associated with lipid rafts $[22,23]$, but their detailed function is unclear. Flotillins are characterized by the SPFH domain of unknown function and extended heptad repeat regions. Recently, flotillin-like proteins FloT and YqfA have been implicated in the clustering of a signal transduction protein in the membrane of B. subtilis cells [24], revealing yet another striking parallel between pro - and eukaryotic cells.

In our work, we show that $B$. subtilis dynamin ortholog (termed DynA) plays a role in cell division. DynA and flotillin-like protein FloT synergistically affect cell division and cell morphology, suggesting that lipid raft formation and dynamin-driven membrane modification are important for cytokinesis and cell shape maintenance in bacteria.

\section{Results}

\section{DynA plays a role in cell division}

We deleted the $d y n A(y p b R)$ gene by long flanking sequence homology PCR, such that only the first and last $100 \mathrm{bp}$ of the gene remained within the chromosome, disrupted by a tet cassette. We also generated a truncated version of $d y n A$ through the insertion of a plasmid into the $d y n A$ gene, driving the downstream gene with a xyloseinducible promoter. Mutant cells grew with a doubling time indistinguishable from that of wild type cells, and contained nucleoids of normal appearance (Figure 1B). However, 5.5\% of the cells showed double septa/mini cells (Figure 1B), which are never observed in wild type cells (Figure 1A). Additionally, $2.5 \%$ of mutant cells were larger than $5.5 \mu \mathrm{m}$ (Figure 1C), while only $0.5 \%$ of wild type cells reach this size (250 cells measured for each strain). In contrast to e.g. a deletion of sftA, encoding for a DNA translocase that couples late states of chromosome segregation and cell division [25,26], DNA was never observed to be trapped in a 

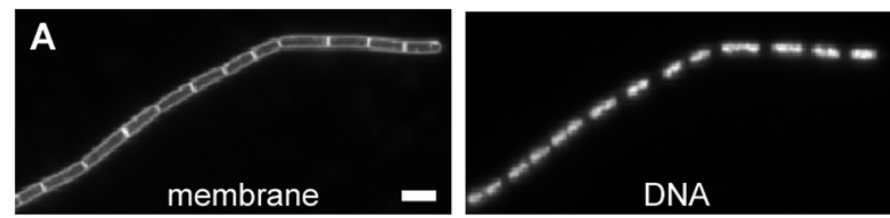

wild type

$\Delta d y n A$

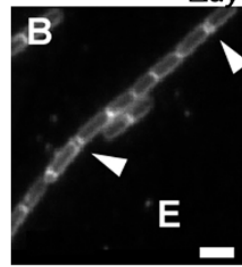

membrane

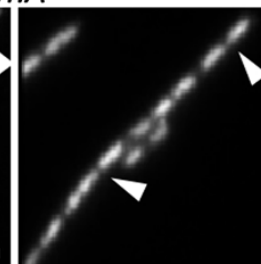

DNA

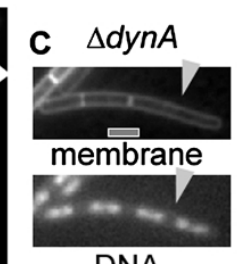

DNA

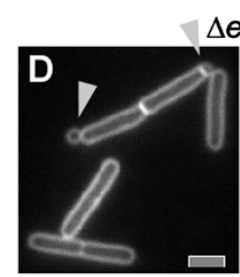

membrane

$\Delta e z r A$

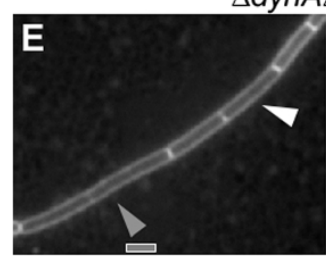

membrane

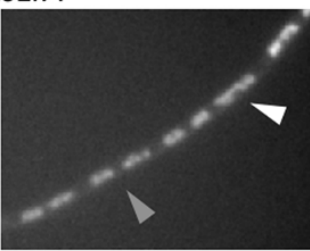

DNA

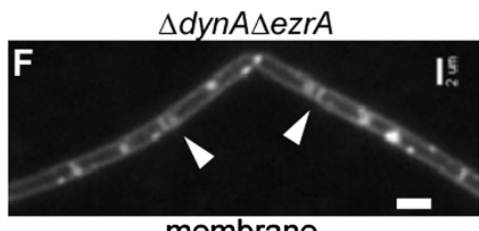

membrane
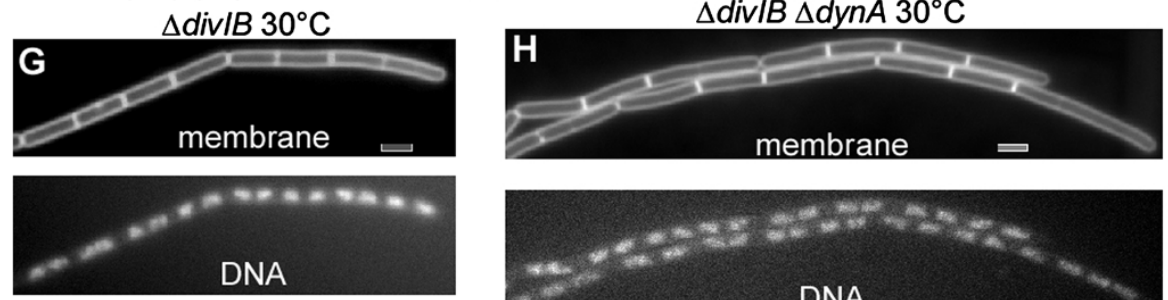

$\triangle$ divlB $42^{\circ} \mathrm{C}$

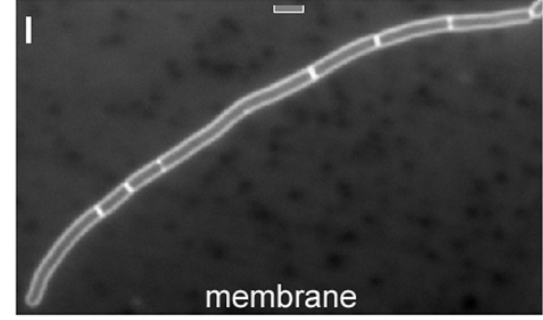

membrane
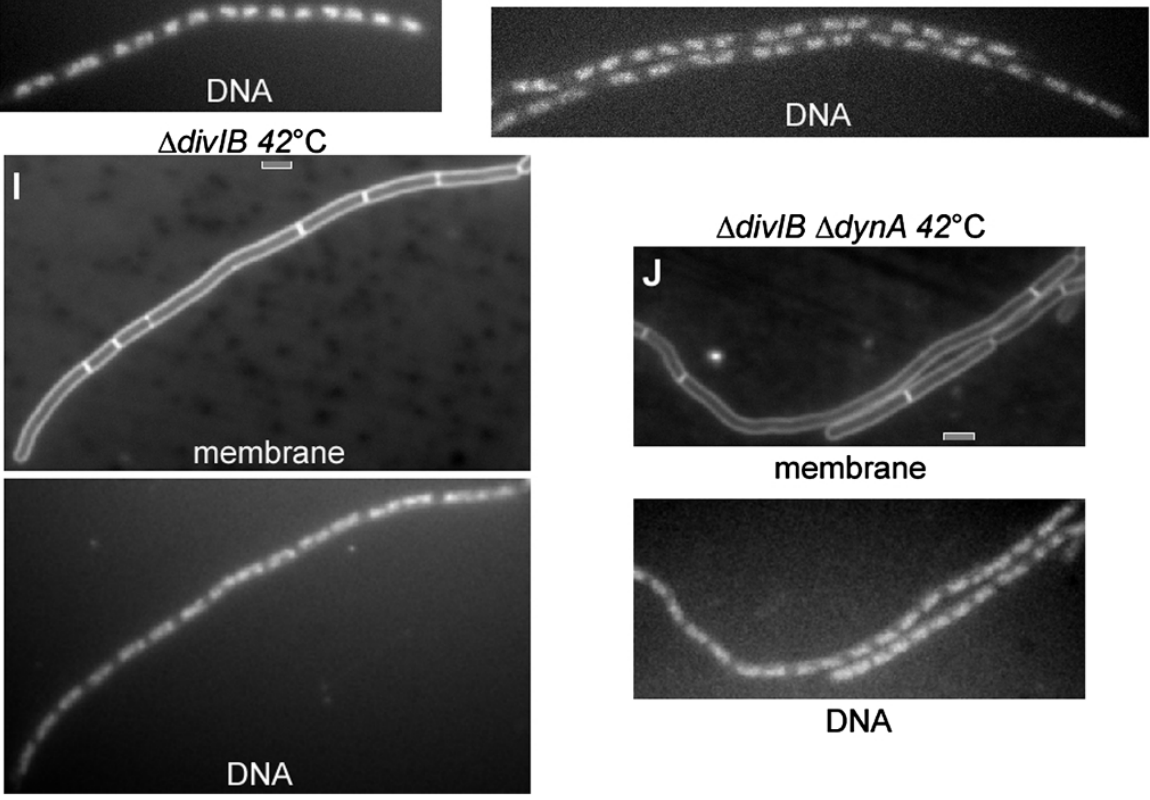

membrane

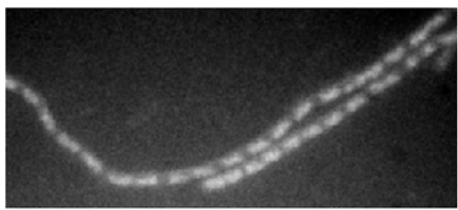

DNA

Figure 1 Phenotypes of exponentially growing wild type (PY79) or mutant Bacillus subtilis cells. A) Wild type cells, B) dynA (ypbR) mutant cells, white triangles indicate double septa, C) dynA (ypbR) mutant cells, grey triangle indicates highly elongated cell, D) ezrA mutant cells, E) ezrA/ dynA double mutant cells, F) ezrA/dynA double mutant cells, white triangles indicate double septa, $\mathbf{G}$ ) divlB mutant cells grown at $\left.30^{\circ} \mathrm{C}, \mathbf{H}\right) \operatorname{div}(B /$ dynA double mutant cells grown at $30^{\circ} \mathrm{C}$, I) diviB mutant cells grown at $42^{\circ} \mathrm{C}, \mathbf{J}$ ) diviB/dynA double mutant cells grown at $42^{\circ} \mathrm{C}$. White or grey bars $2 \mu \mathrm{m}$. 
closed division septum in dynA mutant cells. Therefore, chromosome segregation occurs normally in the mutant cells, but cell division is noticeably defective.

We wished to investigate the effect of a combination of the $d y n A$ deletion with that of a protein known to be important for an initial step in cell division. EzrA is a regulator of FtsZ, and therefore acts at a very early time point during cell division. The deletion of ezrA leads to the generation of elongated cells, to the formation of double septa and mini cells in rich medium [27]. In minimal medium used in this study, ezrA mutant cells were elongated, and formed mini cells (9\%), but did not show any double septa (Figure 1D). Interestingly, ezrA $d y n A$ double mutant cells were more elongated than ezrA single mutant cells (Figure 1E), and contained more double septa than both single mutants (Figure 1F). Double mutant cells measured on average $5.16 \pm 0.5 \mu \mathrm{m}$ versus $4.07 \pm 0.45 \mu \mathrm{m}$ for ezrA mutant cells, and contained double septa in $15 \%$ of the cells versus $5 \%$ in dynA single mutant cells (with 200 cells measured for each strain from 2 independent experiments). Occasionally, long ezrA $d y n A$ double mutant cells showed a single condensed or decondensed nucleoid indicating a segregation defect, but this referred only to a subpupulation of long cells (Figure 1E, white triangle). Thus, the increase in cell length is largely due to an effect on cell division. These data suggest that EzrA and DynA affect two distinct steps early in cell division, each of which contributes to efficient cell division, because all phenotypes are exacerbated by the loss of both proteins.

We also tested if the $d y n A$ deletion is affected by the deletion of a gene involved in a later step of cell division. We used $\operatorname{divIB}$ mutant cells, which show a pronounced defect in cell division when they are shifted from 30 to $42^{\circ} \mathrm{C}$. DivIB is a component of the DivIC/FtsL complex, which is recruited to the $\mathrm{Z}$ ring with a marked delay to the initial $\mathrm{Z}$ ring formation $[17,28,29]$. Indeed, when $\operatorname{divIB}$ mutant cells were shifted to the higher temperature, cells elongated markedly (compare Figure 1G and 1I), which was also true for $d y n A \operatorname{divIB}$ double mutant cells, whose length could not easily be distinguished by eye from the $\operatorname{divIB}$ single mutant strain, neither at $30^{\circ} \mathrm{C}$ (Figure $1 \mathrm{H}$ ) nor at $42^{\circ} \mathrm{C}$ (Figure 1J). We measured average cell length for 140 to 150 cells for each strain and for each growth temperature, from 3 independent experiments. The average cell length of divIB mutant cells was $4.03 \mu \mathrm{m}$ (1.4 $\mu \mathrm{m}$ standard deviation, SD) at $30^{\circ} \mathrm{C}$ and $5.15 \mu \mathrm{m}$ $(4.9 \mu \mathrm{m} \mathrm{SD})$ at $42^{\circ} \mathrm{C}$, while that of $d y n A \operatorname{divIB}$ mutant cells was $3.9 \mu \mathrm{m}(1.2 \mu \mathrm{m} \mathrm{SD})$ at $30^{\circ} \mathrm{C}$ and $6.18 \mu \mathrm{m}(5.15 \mu \mathrm{m}$ $\mathrm{SD})$ at $42^{\circ} \mathrm{C}$. Average cell length of $d y n A$ mutant cells at $42^{\circ} \mathrm{C}$ was $3.75 \mu \mathrm{m}(1.1 \mu \mathrm{m} \mathrm{SD})$. The high standard deviation at $42^{\circ} \mathrm{C}$ stems from the fact that a considerable number of cells were extremely long (up to $25 \mu \mathrm{m}$ ), while most cells had a size below $5 \mu \mathrm{m}$. To account for this, we grouped cells into three categories: cells below $5.5 \mu \mathrm{m}$, cells between 5.5 and $10 \mu \mathrm{m}$, and cells above $10 \mu \mathrm{m}$. For divIB single mutant cells, $6.3 \%$ of the cells were above $5.5 \mu \mathrm{m}$ long, and $0.7 \%$ above $10 \mu \mathrm{m}$ at $30^{\circ} \mathrm{C}$, while at $42^{\circ} \mathrm{C}, 19 \%$ were above $5.5 \mu \mathrm{m}$ and $8 \%$ above $10 \mu \mathrm{m}$. At $30^{\circ} \mathrm{C}, 8.5 \%$ of double mutant cells were above $5.5 \mu \mathrm{m}$ and $1.5 \%$ above $10 \mu \mathrm{m}$, and at $42^{\circ} \mathrm{C}, 34 \%$ were above $5.5 \mu \mathrm{m}$ and $12 \%$ above $10 \mu \mathrm{m}$ (Table 1). Thus, the fraction of double mutant cells was higher in each of the "large cell" categories compared with the single divIB mutant cells. Single and double mutant cells contained normally segregated nucleoids (Figure 1G-J), showing that cell elongation is not an effect of delayed or blocked chromosome segregation. These data show that the deletion of a late cell division gene also exacerbates the $d y n A$ phenotype, showing that DynA does not only affect a step in cell division that is specific to the activity of EzrA.

\section{DynA co-localizes with FtsZ and affects the formation of the $Z$ ring}

We generated a $d y n A(y p b R)-y f p$ fusion that was integrated into the original gene locus. Cells expressing DynA-YFP did not show any double septa, or highly elongated cells, indicating that the fusion can functionally replace the wild type protein and/or any of the possible post-translationally modified versions of DynA. Western blot analysis showed that full length DynA-YFP is expressed at extremely low levels, as well as a C-terminal fragment of $27 \mathrm{kDa}$ and several smaller fragments (Figure 2, note that YFP is $28 \mathrm{kDa}$, giving rise to a band of $55 \mathrm{kDa}$ ). The $27 \mathrm{kDa}$ band appeared at roughly the same level as full length protein. It is unclear if DynA is subject to rapid degradation through proteolysis or if the protein is proteolytically processed. Processing of DynA into two dynamin-like proteins (it consists of two fused dynamin modules) would give rise to 62 to $63 \mathrm{kDa}$ sized proteins, which would be $90 \mathrm{kDa}$ when fused to YFP. This is not the case according to the Western blot analysis. It is unclear if the truncation product is generated through the YFP fusion construct, or also occurs for wild type DynA. Therefore, localization studies must be viewed in light of the caveat that the truncation product may confer some level of DynA activity.

Table 1 Distribution of cell length in single and double mutant cells

\begin{tabular}{lccc}
\hline & $<\mathbf{5 . 5} \boldsymbol{\mu m}$ & $>\mathbf{5 . 5} \boldsymbol{\mu m}<\mathbf{1 0} \boldsymbol{\mu m}$ & $>\mathbf{1 0} \boldsymbol{\mu m}$ \\
\hline$\Delta \operatorname{div} \mid B 30^{\circ} \mathrm{C}$ & $93 \%$ & $6.3 \%$ & $0.7 \%$ \\
$\Delta \operatorname{dyn} A \Delta \operatorname{div} \mid B 30^{\circ} \mathrm{C}$ & $90 \%$ & $8.5 \%$ & $1.5 \%$ \\
$\Delta \operatorname{div} \mid B 42^{\circ} \mathrm{C}$ & $73 \%$ & $19 \%$ & $8 \%$ \\
$\Delta \operatorname{dyn} A \Delta \operatorname{div} \mid B 42^{\circ} \mathrm{C}$ & $64 \%$ & $34 \%$ & $12 \%$ \\
\hline
\end{tabular}




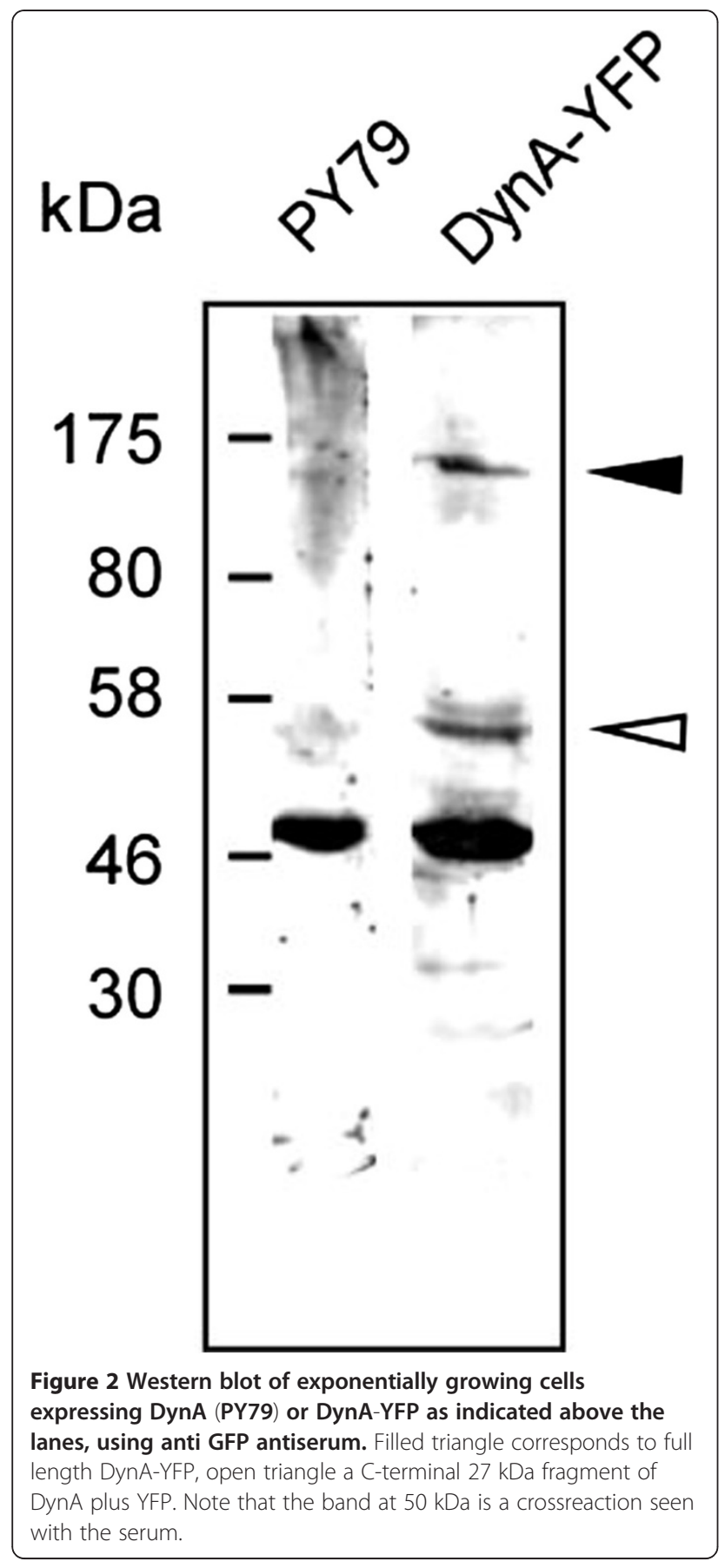

DynA-YFP localized to the cell center in exponentially growing cells (Figure 3A), and formed one or two foci at irregular places along the membrane in $15 \%$ of the cells (Figure 3B, 200 cells analyzed). Thus, in contrast to e.g. the membrane protein $\mathrm{MreC}$, which localises as distinct foci throughout the membrane (Figure 3C, note that there are two adjacent membranes at the division septum), DynA is clearly highly enriched at the future division site. Indeed, DynA-YFP co-localized with FtsZ-
CFP (Figure 3A); clear DynA-YFP fluorescence was seen at $85 \%$ of FtsZ-CFP rings, and $15 \%$ of $\mathrm{Z}$ rings were devoid of detectable DynA-YFP fluorescence (250 cells analysed), which, however, was extremely faint. Many cells contained DynA-YFP foci rather than ring-like structures (Figure $3 \mathrm{~A}$, indicated by white triangle). These data indicate that DynA is recruited to the $\mathrm{Z}$ ring, possibly at an early time point during cell division.

During stationary phase, many cells showed multiple DynA-YFP foci, while most cells $(60 \%)$ did not reveal any focus (Figure 3D). The observed foci were generally proximal to the cell membrane (Figure 3D), suggesting that DynA is largely a membrane-associated protein, although no obvious transmembrane segment is predicted by standard secondary structure prediction. Thus, DynA is associated with the cell division machinery in growing cells, in agreement with the observed phenotype of the $d y n A$ deletion, and remains membrane-associated in non-growing cells.

The apparent effect on cytokinesis prompted us to study the localization of FtsZ in dynA mutant cells. Although $\mathrm{Z}$ rings were normally positioned at mid cell in most $d y n A$ mutant cells, several abnormal morphologies of $\mathrm{Z}$ rings were observed: a) $\mathrm{Z}$ rings that appeared to be an open helix (Figure 3E, left panel), b) Z rings that were brighter on one side (Figure 3E, right panel), c) double septa (not shown) and d) missing rings in very large cells $(>4 \mu \mathrm{m}$, Figure $3 \mathrm{E}$, right panel), which in wild type cells invariably contain $\mathrm{Z}$ rings. These aberrant structures were seen in about $15 \%$ of $d y n A$ mutant cells (180 cells analysed), indicating that DynA has an effect on the formation of a proper FtsZ ring, directly or indirectly, and that the defect in cell division arises largely through the loss of this function.

A synthetic defect in cell division, cell shape maintenance and motility for dynamin and flotillin double mutant cells Eukaryotic membranes appear to have an asymmetric distribution of lipids, and specific proteins associated with the so-called lipid rafts. Flotillins are a divergent membrane protein family associated with lipid rafts, and are characterized by the SPFH domain of unknown function and extended heptad repeat regions [30]. B. subtilis flotillin-like proteins FloT and YqfA are involved in the clustering of a signal transduction protein in the membrane [24], and in the timing of initiation of sporulation [31]. Eukaryotic flotillin proteins are involved in clathrin-independent endocytosis, and in other processes, where membrane bending is of importance [32]. We reasoned that lipid rafts and bacterial dynamin may synergistically facilitate cell division, and therefore combined floT and $d y n A$ deletions. Strikingly, double mutant cells were highly elongated and showed a strong defect in cell shape maintenance (Figure 4A). 

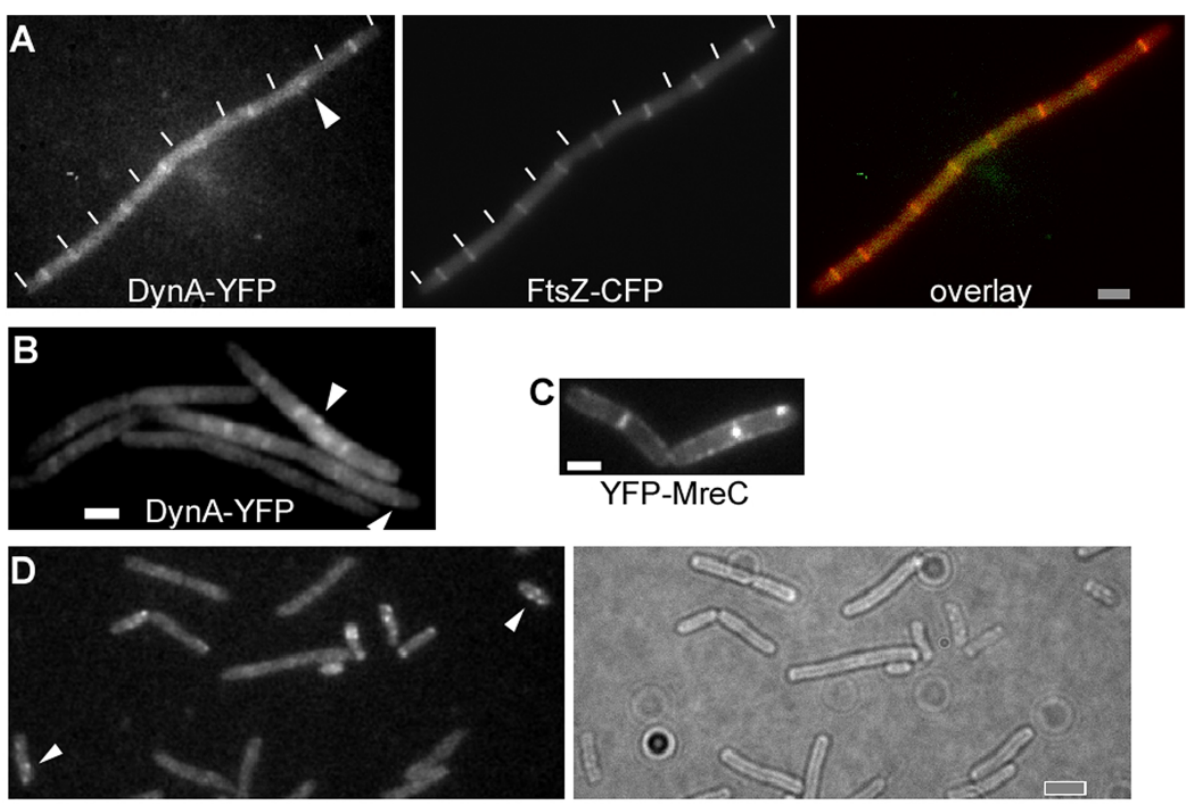

\section{DynA-YFP}

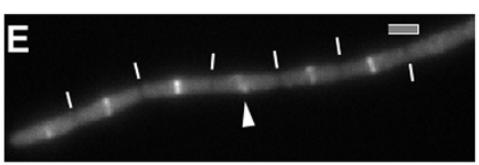

Nomarski

FtsZ-CFP
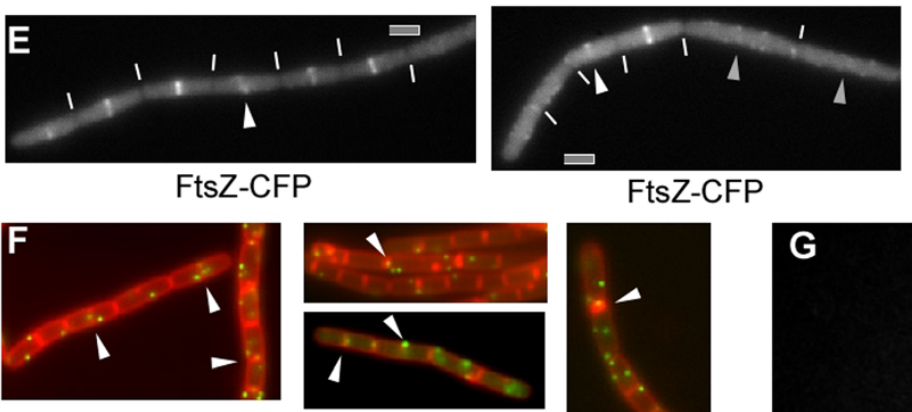

FloT-YFP/membrane

H

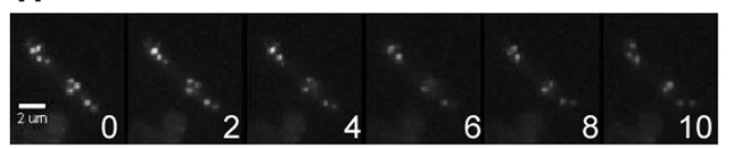

FloT-YFP $\triangle d y n A$

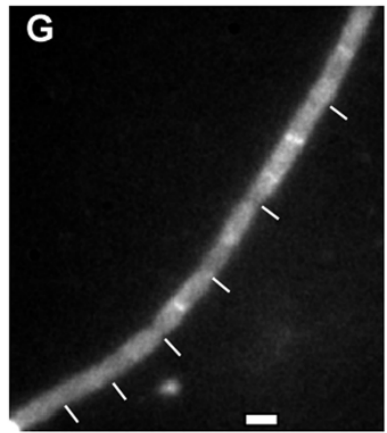

DynA-YFP $\triangle f$ floT

Figure 3 Localization of DynA, FtsZ, FloT and MreB. A-B) Growing wild type cells expressing DynA-YFP and FtsZ-CFP, white lines indicate septa between cells, overlay: FtsZ-CFP in red, DynA-YFP in green, C) cells expressing YFP-MreC, D) stationary phase cells expressing DynA-YFP, white triangles indicate membrane-proximal foci, E) dynA (ypbR) mutant cells expressing FtsZ-CFP, white triangles indicate asymmetric FtsZ rings, grey triangles large cells lacking FtsZ rings but instead containing membrane-proximal accumulations of FtsZ-CFP: white lines indicate septa between cells, F) wild type cells expressing FloT-YFP, overlay with membranes (red) and FloT-YFP (green), G) floT mutant cells expressing DynAYFP. H) dynA mutant cells expressing FloT- YFP, time lapse with images taken every $2 \mathrm{~s}$. White or grey bars $2 \mu \mathrm{m}$.

Many cells were bent and had an irregular width, and a considerable fraction could reach a size of $12 \mu \mathrm{m}$. Frequently, cells showed aberrant membrane staining (Figure 4A), including large membrane perturbations. Although nucleoids were irregularly positioned, we did not observe any anucleate cells. In contrast to an smc mutant strain, in which chromosomes are highly decondensed and fill the entire cytoplasm (in which nucleoid occlusion blocks cell division [33]), floT/yprB double mutant cells contained many DNA-free sites in which nucleoid occlusion would not block division. However, cells were highly filamentous, suggesting that FloT and DynA synergistically affect cell division, in addition to an effect on rod-shape cell elongation. In agreement with the cytological data, the double mutant strain grew much slower than the wild type, and had a highly extended lag phase (Figure 5). Double mutant cells grew with a doubling time of $73 \mathrm{~min}$ versus $44 \mathrm{~min}$ at $37^{\circ} \mathrm{C}$ 


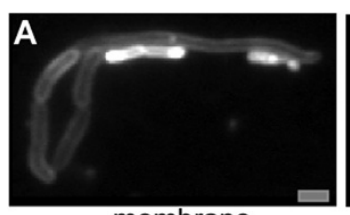

$$
\text { membrane }
$$

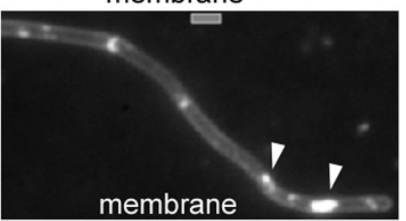

membrane

B
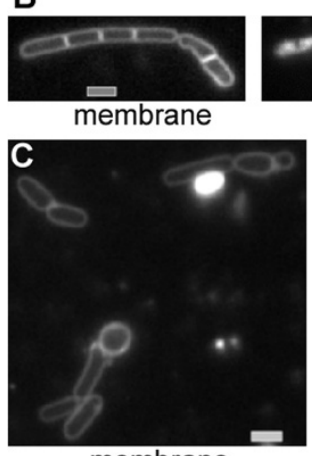

membrane

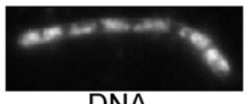

DNA

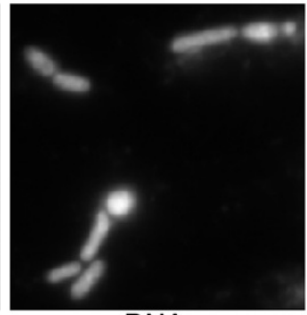

DNA
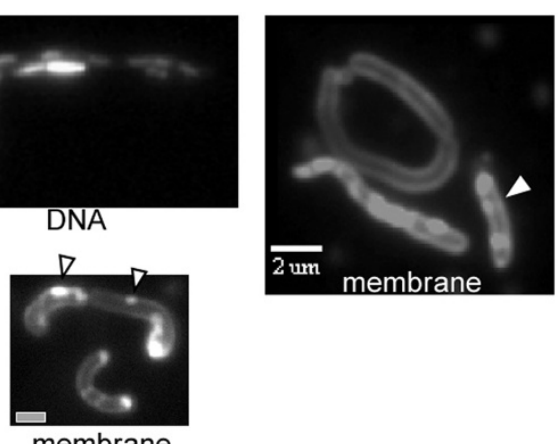

membrane

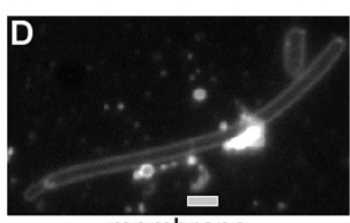

membrane

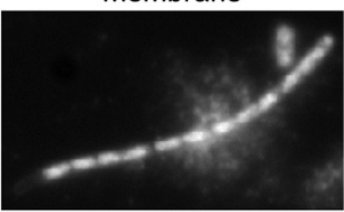

DNA

Figure 4 Phenotypes of exponentially growing double mutant $\boldsymbol{B}$. subtilis cells. A) dynA/floT double mutant cells (note that membrane staining is highly heterogeneous between cells), white triangles indicate membrane abnormalities, B) mreB mutant cells grown in high magnesium medium, C-D) dynA/mreB double mutant cells growing in high magnesium medium. White or grey bars $2 \mu \mathrm{m}$.

for wild type cells or for both single mutant strains, showing that growth was strongly compromised by the loss of both membrane-associated proteins.

Based on its ability to tubulate membranes in vitro $[11,13]$, DynA may facilitate membrane invagination through a mechanical bending of the membrane, while FloT may be important to generate a local environment favoring membrane curvature and/or recruitment of cell division proteins. In agreement with its function in lipid raft formation, a functional FloT-YFP fusion formed many discrete foci at the cell membrane [34] (Figure 3F). FloTYFP was previously shown to move along random paths within or adjacent to the membrane [34]. These findings imply that due to the random movement, FloT would also be frequently present at mid cell, which indeed was shown to be the case by colocalization of FloT-YFP with membrane stain FM4-64 [34].To obtain a better idea about the extent of colocalization of FloT with the septal membrane, we quantified the number of FloT-YFP foci between cells. Indeed, $26 \%$ of FloT-YFP foci colocalized with the septal/

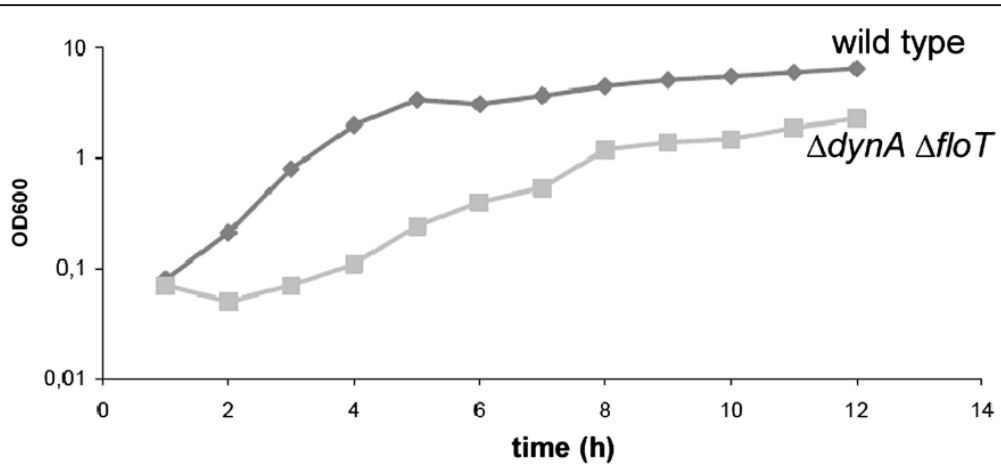

Figure 5 Growth curve of wild type cells (diamonds) or of dynA/floT double mutant cells (squares) growing in S750 minimal glucose medium containing $0.1 \%$ casamino acids at $37^{\circ} \mathrm{C}$. Data are means from four independently growing cultures. 
polar membrane (184 foci analysed), or in other words, $22 \%$ of the cells had FloT-YFP fluorescence at the septum (Figure 3F, green FloT-YFP foci, red membrane) (148 cells analysed from 3 independent experiments), showing that FloT is present at sites of cell division in a large fraction of the cells; even more cells contained FloT-YFP foci close to the cell centre.

To investigate if one protein affects the localization of the other, we localized DynA-YFP in delta floT (yuaG) mutant cells. The localization pattern was indistinguishable from that of wild type cells (Figure 3G). Conversely, the absence of DynA did not visibly alter the localization pattern and dynamics of FloT-YFP (Figure 3H), showing that the proteins do not affect each other's localization within the cell membrane and that they are not functionally linked.

\section{Synthetic phenotype of a dynA mreB double mutant strain}

Because floT dynA double mutant cells had a highly disturbed cell shape, we investigated the effect of a $d y n A$ deletion in combination with an $m r e B$ deletion. MreB is essential for the maintenance of rod shape in many bacteria, and the depletion of MreB leads to the generation of round cells that eventually lyse $[20,35]$. However, $m r e B$ mutant cells can be grown in a medium containing a high concentration of magnesium [36], in which they show only a mild cell shape defect (Figure 4B). When grown under high magnesium conditions, a majority of $d y n A$ mreB double mutant cells showed a synthetic cell shape as well as division defect. A large fraction of cells was round or club-shaped, which was not observed for single mutant cells (Figure 4C). A second (smaller) fraction of cells was highly elongated (> $15 \mu \mathrm{m}$ length), and many of these cells showed an irregular cell diameter along the length of the filaments (Figure 4D). In contrast to $d y n A$ floT double mutant cells, dynA mreB double mutants did not show membrane-abnormalities, indicating that these occur specifically due to the loss of dynamin and flotillinlike proteins, and not to a general alteration of cell morphology. Many dynA mreB double mutant cells contained decondensed chromosomes, but also contained segregated nucleoids, between which no septum was detectable, in spite of the excessive length of the cells (Figure 4D). In total, more than $90 \%$ of all double mutant cells showed a cell shape defect, while only $18 \%$ of the mreB single mutant cells showed a clear change in cell morphology (280 cells analysed). Therefore, DynA also plays a role in cell shape maintenance that is exacerbated by the loss of MreB.

To find out if DynA may have an effect in the formation of MreB filaments, as it has on the formation of the FtsZ ring, we visualized YFP-MreB in $d y n A$ mutant cells. Indistinguishably from wild type cells, YFPMreB formed filamentous structures in mutant cells, which showed wild type-like remodeling (data not shown), showing that DynA itself does not directly affect the MreB cytoskeleton.

\section{Self assembly of DynA and of FloT at the membrane in a heterologous cell system}

We wished to obtain information on the intrinsic properties of DynA, and therefore expressed the YFP fusion protein in Schneider S2 cells. These cells from Drosophila flies are highly diverged from the bacterial system, and because DynA displays less than 20\% sequence identity with dynamin, it is highly unlikely that DynA has any specific interactors in S2 cells, or interacts with dynamin itself. Early after transfection, DynA-YFP assembled at internal membrane systems as well as underneath the cell membrane, suggesting that it has intrinsic membrane affinity (Figure 6A). After extended expression (6 hours and longer), DynA formed network-like structures at the cell membrane (note that membrane staining does not clearly show the outline of the membrane due to a high internal background, see Figure 6D). These structures resembled tubulated membrane structures, which extended away from the cells (Figure 6B). Non-transfected cells showed tubular membrane extensions at an about 100 fold lower level and only background fluorescence (Figure 6D), and started to form cell extensions 12 hours after deposition on glass slides (a time when they started to move), showing that DynA self assembles underneath the cell membrane and considerably distorts the membrane. In our model, we predict that dynamin distorts the cell membrane inwards during cell division, which is opposite from the orientation of the tubules observed in S2 cells. However, directionality of membrane distortion may be directed by other bacterial factors (e.g. by FtsZ), and tubules may also be caused by overproduction of DynA. In any event, our experiments show that DynA has the ability to induce considerable membrane distortion.

In contrast to DynA, FloT assembled only infrequently at internal membrane systems (occasionally, FloT-YFP was found around the nucleus) but predominantly at the cell membrane (Figure 6C), where it formed differently sized patch structures, as previously reported [34]. Given that FloT has extended coiled coil structures, we cannot exclude that the protein non-specifically interacts with other proteins within the membrane. However, usually, coiled coil interactions are rather specific, so our data indicate that FloT may self-assemble into raft-like structures in a heterologous system that lacks any other bacterial protein. FloT-YFP expressing cells showed very few tubulated membrane structures, verifying that DynA induces strong membrane deformation. 

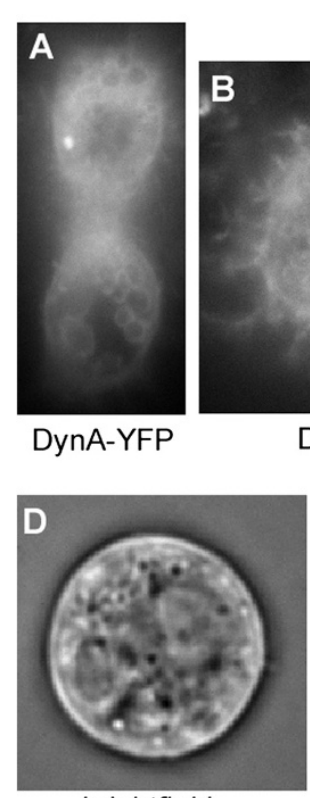

brightfield

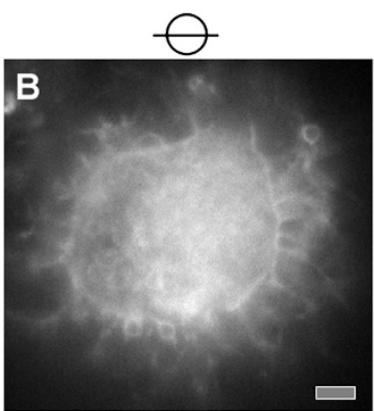

DynA-YFP

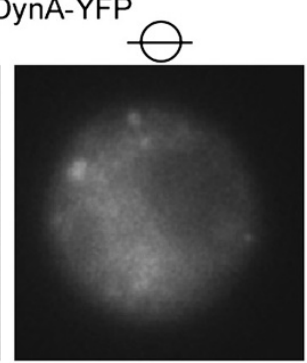

YFP

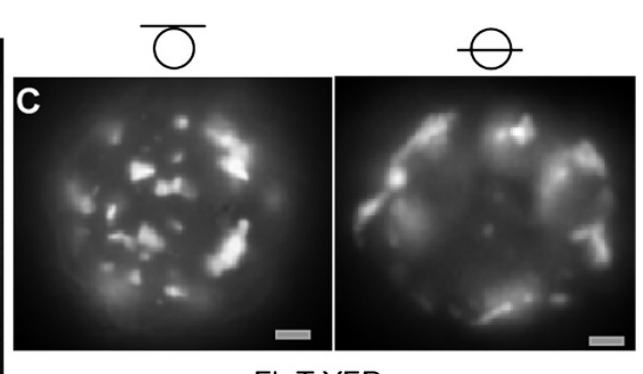

FloT-YFP

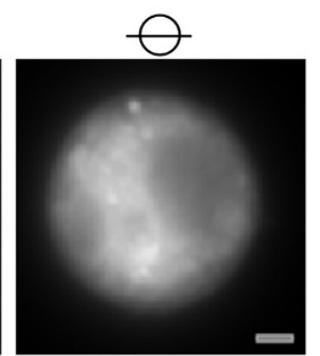

membrane

Figure 6 YFP fluorescence of Drosophila S2 cells expressing fusion proteins. A) cells expressing DynA-YFP early after induction, or B) 6 hours after induction. Shown are planes in the middle of cells, C) S2 cells expressing FloT-YFP, shown is the middle plane or the surface of the cells, as indicated by the lines within the circle. D) Non-transfected cells, the outline can be seen in the bright field channel; membrane stain also shows the outline of cells, but the membrane cannot be distinguished from the background of the cell; panel "YFP" shows background fluorescence in non-transfected cells in the YFP channel. White or grey bars $2 \mu \mathrm{m}$.

\section{Discussion}

Bacterial dynamin-like proteins (BDLPs) have been characterized in vitro, and based on their ability to generate membrane tubulation and membrane fusion in vitro, a role in membrane dynamics [12], e.g. in late steps in cell division [13], has been proposed. However, it has been unclear if BDLPs confer any important role on the physiology of the cell. Through the combination of a $d y n A$ deletion with deletions in two genes involved in cell division, we show that indeed, DynA confers a function during cell division. A single $d y n A$ deletion leads to a very mild defect in $\mathrm{Z}$ ring formation, similar to, but less pronounced than, a deletion in $e z r A$. This is in agreement with our data showing that DynA colocalizes with FtsZ. $85 \%$ of the $\mathrm{Z}$ rings showed DynA-YFP signals (and because of the very weak fluorescence, the actual number could be higher). It has been shown that during spore germination, proteins such as EzrA and FtsA are recruited to the $\mathrm{Z}$ ring during the onset of division, while some proteins (such as DivIc and DivIb) are recruited with a 10 min time delay [17]. Assuming that this delay also applies to exponentially growing cells and not only to germinating cells, DynA might belong to the early cell division proteins, which must be verified by additional experiments. A dynA ezrA double deletion leads to a strongly exacerbated phenotype in cell division, suggesting that like EzrA, a regulator of FtsZ ring formation, B. subtilis dynamin affects an early stage in cell division. However, the combination of a $d y n A$ deletion with a $\operatorname{divIB}$ deletion also leads to a synthetic effect on cell division. DivIB affects a state in division clearly later than the formation of the $\mathrm{Z}$ ring, indicating that the function of DynA in division cannot be correlated with a defined stage in division. In any event, the accumulation of dynamin at the $\mathrm{Z}$ ring underlines the idea that dynamin confers a function during division.

Expression of DynA in a eukaryotic cell system showed that the protein has intrinsic affinity to the cell membrane and can assemble into tubulated structures. However, these pointed outwards of the cells, while the assumed function of dynamin in the bacterial cell would either be an inward bending of the membrane during cell division, or the fusion of membranes as the last step during division. It is likely that DynA needs cofactors for its appropriate function in the bacterium.

Interestingly, the combination of a $d y n A$ deletion with the deletion of a gene encoding for a flotillin-like protein, FloT, also leads to a synthetic defect in cell division. Flotillin proteins are implicated in lipid raft formation in eukaryotic and in prokaryotic cells. Although our experiments do not allow us to make any clear conclusion as to the detailed function of dynamin or flotillin, they show that bacterial dynamin and flotillin proteins play non-redundant functions in membrane dynamics. This is supported by our findings that each mutation does not affect the localization 
of the other protein. We suggest that dynamin is important for the generation of cell curvature, possibly via its putative mechanochemical activity, and likewise flotillin proteins, which may be important to recruit lipids that favour membrane bending. Indeed, there appears to be a link between flotillin in B. subtilis and membrane fluidity [37]. This idea is supported by our finding that DynA can distort the cell membrane in a heterologous cell system, suggesting that DynA may facilitate membrane invagination and/or couple Z-ring formation with membrane invagination. Alternatively, flotillin may be important to facilitate the recruitment of cell division proteins to the $\mathrm{Z}$ ring. In any event, the role of dynamin and flotillins in cell division is not redundant, because of the synthetic effect, and because of their different localization patterns.

The idea that dynamin and flotillin play general roles in membrane dynamics, rather than a role specific for cell division, is supported by three additional findings in our work: firstly, dynamin/flotillin double mutant cells grow much slower than wild type or single mutant cells, while cells depleted for FtsZ initially grow as fast as wild type cells. Thus, additional effects on cell growth are apparent in double mutant cells. Secondly, dynA floT double mutant cells also show a strong defect in cell morphology, and thirdly, the lack of the cytoskeletal element MreB in addition to the loss of dynamin function exacerbates the MreB cell shape phenotype. MreB can be deleted in the presence of high concentrations of magnesium (but not in normal medium), and the deletion of $d y n A$ under these conditions leads to a complete loss of rod cell morphology. Thus, dynamin function is also important in the context of maintenance of rod cell shape. DynA is not epistatic with MreB, showing that DynA does not act on cell morphology via the MreB cytoskeleton. Using fluorescence microscopy, we clearly identified DynA molecules along the lateral cell wall, away from the cell centre, which may be involved in functions affecting cell morphology.

\section{Conclusion}

In toto, in our work, we uncover a role for DynA, the Bacillus subtilis ortholog of eukaryotic dynamin and of cyanobacterial BDLP, in cell division and in cell shape maintenance, and reveal a genetic link between bacterial dynamins and flotillins. We provide evidence that dynamin can self-assemble at the membrane and lead to membrane distortion in the absence of any bacterial cofactor. It is important to note that the lack of dynamin and of flotillin, or of dynamin and MreB, a gene involved in cell shape maintenance, results in various defects in the physiology of a bacterial cell, so the function of dynamin is not restricted to cell division. The data suggest that lipid rafts and dynamin-mediated membrane distortion play a synergistic role in a variety of membrane-associated assembly processes, the molecular nature of which needs to be further investigated.

\section{Methods}

\section{Bacterial strains and media}

Bacillus strains (Table 2) were grown in LB medium or, for microscopy, in S750 defined medium [38], which was complemented with $0.004 \%(\mathrm{w} / \mathrm{v})$ casamino acids. Selection pressure with appropriate antibiotics was always kept when growing different strains. Cells were grown to exponential phase at $30^{\circ} \mathrm{C}$.

For the deletion of $d y n A(y p b R)$, the first $100 \mathrm{bp}$ of $d y n A$ plus 900 bp upstream were amplified via PCR, and likewise the last $100 \mathrm{bp}$ plus the following $900 \mathrm{bp}$. Inner primers contained $18 \mathrm{bp}$ of homology to the tet cassette, which was amplified together with flanking sequences in a second PCR reaction. The resulting $4000 \mathrm{bp}$ fragment was used for transformation of PY79 wild type cells, selecting for tetracycline (tet) resistance, giving rise to HW2 (dynA::tet). As an alternative strategy, $500 \mathrm{bp}$ internal of $d y n A$ (starting at bp 1480) were amplified and cloned into pMutin, using HindIII and EcoRI restriction sites. PY79 cells were transformed with plasmid DNA, selecting for Mls resistance, giving rise to a DynA truncation missing the last 500 amino acids.

For the generation of a dynA floT double mutant strain, strain DML1541 $\Delta$ floT (yuaG) (in frame deletion

\section{Table 2 Strains used in this study}

\begin{tabular}{|c|c|c|}
\hline PY79 & wt & \\
\hline HW2 & dynA::tet & This study \\
\hline HW3 & dynA::pMutin & This study \\
\hline FD249 & dynA::tet $\Delta$ floT(in frame deletion) & This study \\
\hline HW1 & dynA-yfp $\left(\mathrm{cm}^{\mathrm{R}}\right)$ & This study \\
\hline HW4 & dynA-yfp $\left(\mathrm{cm}^{\mathrm{R}}\right)$ ftsZ-cfp $\left(\right.$ spec $\left.^{\mathrm{R}}\right)$ & This study \\
\hline HW5 & dynA:.:tet ftsZ-cfp $\left(\right.$ spec $\left.^{R}\right)$ & This study \\
\hline HW6 & dynA::tet yfp-mreB $\left(\right.$ spec $\left.^{R}\right)$ & This study \\
\hline FD295 & floT-yfp $\left(\mathrm{cm}^{\mathrm{R}}\right)$ & [34] \\
\hline FD258 & dynA::tet floT-yfp $\left(\mathrm{cm}^{\mathrm{R}}\right)$ & This study \\
\hline 3725 & $\Delta m r e B$ (in frame deletion) & [36] \\
\hline HW7 & dynA:-tet $\Delta m r e B$ & This study \\
\hline HW8 & dynA::tet ezrA:.spec & This study \\
\hline $\mathrm{HIHO} 114$ & $\Delta f l o T$ in frame deletion & Gift from M. Hinderhofer \\
\hline BS1059 & $P_{x y l} f t s Z-c f p\left(s p e c^{R}\right)$ & {$[39]$} \\
\hline$J S 12$ & $P_{\text {xyl }}$ yfp-mreB $\left(\operatorname{spec}^{R}\right)$ & [40] \\
\hline FG375 & ezrA::spec & [41] \\
\hline \multicolumn{3}{|c|}{ Plasmids used for transfection } \\
\hline pFD1 & Expression vector for $\mathrm{S} 2$ cells & {$[42]$} \\
\hline pFD239 & floT-yfp in pFD1 & {$[34]$} \\
\hline pHW1 & dynA-yfp in pFD1 & This study \\
\hline
\end{tabular}


of $y u a G$, kind gift from M. Hinderhofer, University of Konstanz) was transformed with chromosomal DNA from strain HW2, selecting for tet resistance. For the generation of a C-terminal YFP fusion to DynA, the last 500 bp of $d y n A$ were amplified by PCR and were closed into pSG1164YFP [43] using ApaI and EcoRI restriction sites. PY79 cells were transformed with the resulting plasmid, which integrated at the $d y n A$ locus via single crossover integration (this was verified by PCR using a pair of primers that binds within the $y f p$ gene and upstream of the $500 \mathrm{bp}$ used for integration). Expression of full length DynA-YFP was verified by Western blotting. For simultaneous visualization of DynA and of FtsZ, strain HW1 (DynA-YFP) was transformed with chromosomal DNA from strain BS1059 [39], in which FtsZ-CFP is expressed from a xylose inducible fusion at the amylase locus. The resulting colonies were obtained through selection on spectinomycin containing plates. For the localization of FtsZ-CFP or of YFP-MreB in $d y n A$ mutant cells, strain BS1059 or JS12 was transformed with chromosomal DNA from strain HW2, respectively.

To visualize FloT-YFP in the absence of DynA, strain HW2 $(\Delta d y n A)$ was transformed with chromosomal DNA of strain FD295 $(f l o T-y f p)$. To create a dynA mreB double deletion, strain 3725 [36] $(\triangle m r e B)$ was transformed with chromosomal DNA of strain HW2 $(\triangle d y n A)$ and incubated at $25^{\circ} \mathrm{C}$ using $\mathrm{PAB} / \mathrm{SMM}$ agar [44]. The ezrA dynA double deletion was created by transformation of strain HW2 $(\triangle d y n A)$ with chromosomal DNA of strain FG375 (kind gift from F. Gueiros-Filho, University of São Paulo, Brasil).

The plasmids used for S2 cell transfection were created by cloning the complete coding sequence of DynA or of FloT into the vector pFD1 [45], using KpnI and XhoI or ApaI and ClaI, respectively.

\section{Schneider cell culture and transient transfection}

D. melanogaster S2 Schneider cells were grown in Schneider's Drosophila medium (Lonza Group Ltd.) supplemented with $5-10 \%(\mathrm{v} / \mathrm{v})$ fetal calf serum (FCS) at $25^{\circ} \mathrm{C}$ without addition of $\mathrm{CO}_{2}$. Cells were passaged every 2 to 3 days to maintain optimal growth. For transfection, S2 cells were spread in a 6-well plate at $1 \times 10^{6}$ per well in $3 \mathrm{ml}$ medium containing $5 \%$ FCS. Supercoiled plasmids $(0.3 \mu \mathrm{g}$ of each plasmid) were complexed with lipid (10 $\mu \mathrm{l}$ FuGENE HD reagent, Roche) in $200 \mu \mathrm{l}$ serum-free medium. The complex was incubated at room temperature for $15 \mathrm{~min}$, filled up with serum-free medium to $1 \mathrm{ml}$ and then added to cells from which the growth medium was removed (cells were washed $1 \times$ with serum-free medium). After $18 \mathrm{hrs,} \mathrm{the} \mathrm{complex}$ suspension was removed and replaced by $3 \mathrm{ml}$ of medium containing $10 \% \quad(\mathrm{v} / \mathrm{v})$ FCS. After further incubation for $24 \mathrm{~h}$, the production of the proteins was induced by adding $\mathrm{CuSO}_{4}$ to a final concentration of $1 \mathrm{mM}$.

\section{Image acquisition}

Fluorescence microscopy was performed on an Olympus AX70 microscope with a Cool Snap ES2 camera (Photometrics), TIRF microscopy was performed on an inverted Zeiss Axioobserver microscope with a TIRF incorporation from Visitron (Munich), and an Evolve EMCCD camera (Photometrics). Cells were mounted on thin agarose pads (1\% w/v prepared in $\mathrm{S}_{50}$ minimal medium) on an object slide. DNA was stained with 4', 6-diamidino-2-phenylindole (DAPI; final concentration $0.2 \mathrm{ng} / \mathrm{ml}$ ), membranes with FM4-64 (Molecular Probes). Images were processed with Metamorph software.

\section{Competing interests}

There are no financial or non-financial competing interests concerning this publication. The article processing charge was funded by the German Research Foundation (DFG) and the Albert Ludwigs University Freiburg in the funding programme Open Access Publishing. The University does not gain any financially from this publication.

\section{Authors' contributions}

FD generated genetic constructs and strains, performed most image acquisitions, evaluated data and helped writing the manuscript. HW generated genetic constructs and strains, and performed several microscopy experiments. FD and HW performed growth experiments. MS constructed strains concerning the divlb mutation and performed the related experiments. PLG conceived of the study and wrote the manuscript. PLG, FD, HW and MS evaluated data. All authors read and approved the final manuscript.

\section{Acknowledgments}

We thank Marcus Hinderhofer of the University of Konstanz for the gift of the yuaG (floT) in frame deletion strain, and Joel Defeu Soufo of the University of Freiburg for the gift of mreB strains. This work was supported by the Deutsche Forschungsgemeinschaft (IRTG 1478).

\section{Author details}

'Mikrobiologie, Fachbereich für Biologie, University of Freiburg, Schänzlestraße 1, Freiburg 79104, Germany. ${ }^{2}$ LOEWE Center for Synthetic Microbiology, SYNMIKRO, Philipps-University Marburg, Marburg 35043, Germany. ${ }^{3}$ BIOSS Centre for Biological Signalling Studies, University of Freiburg, Schänzlestr. 18, Freiburg 79108, Germany.

Received: 21 June 2012 Accepted: 7 December 2012 Published: 19 December 2012

\section{References}

1. Hinshaw JE: Dynamin and its role in membrane fission. Annu Rev Cell Dev Biol 2000, 16:483-519.

2. Osteryoung KW, Nunnari J: The division of endosymbiotic organelles. Science 2003, 302(5651):1698-1704.

3. Low HH, Lowe J: Dynamin architecture-from monomer to polymer. Curr Opin Struct Biol 2010, 20(6):791-798.

4. Praefcke GJ, McMahon HT: The dynamin superfamily: universal membrane tubulation and fission molecules? Nat Rev Mol Cell Biol 2004, 5(2):133-147.

5. Song BD, Schmid SL: A molecular motor or a regulator? Dynamin's in a class of its own. Biochemistry 2003, 42(6):1369-1376.

6. Danino D, Hinshaw JE: Dynamin family of mechanoenzymes. Curr Opin Cell Biol 2001, 13(4):454-460.

7. Niemann HH, Knetsch ML, Scherer A, Manstein DJ, Kull FJ: Crystal structure of a dynamin GTPase domain in both nucleotide-free and GDP-bound forms. EMBO J 2001, 20(21):5813-5821. 
8. Baba T, Damke H, Hinshaw JE, Ikeda K, Schmid SL, Warnock DE: Role of dynamin in clathrin-coated vesicle formation. Cold Spring Harb Symp Quant Biol 1995, 60:235-242.

9. Pucadyil TJ, Schmid SL: Conserved functions of membrane active GTPases in coated vesicle formation. Science 2009, 325(5945):1217-1220.

10. Sever S, Damke H, Schmid SL: Dynamin: GTP controls the formation of constricted coated pits, the rate limiting step in clathrin-mediated endocytosis. J Cell Biol 2000, 150(5):1137-1148.

11. Low HH, Lowe J: A bacterial dynamin-like protein. Nature 2006, 444(7120):766-769.

12. Low HH, Sachse C, Amos LA, Lowe J: Structure of a bacterial dynamin-like protein lipid tube provides a mechanism for assembly and membrane curving. Cell 2009, 139(7):1342-1352.

13. Burmann F, Ebert N, van Baarle S, Bramkamp M: A bacterial dynamin-like protein mediating nucleotide-independent membrane fusion. $\mathrm{Mol}$ Microbiol 2011, 79(5):1294-1304.

14. Adams DW, Errington J: Bacterial cell division: assembly, maintenance and disassembly of the Z ring. Nat Rev Microbiol 2009, 7(9):642-653.

15. Rothfield L, Taghbalout A, Shih YL: Spatial control of bacterial division-site placement. Nat Rev Microbiol 2005, 3(12):959-968.

16. Margolin W: FtsZ and the division of prokaryotic cells and organelles. Nat Rev Mol Cell Biol 2005, 6(11):862-871.

17. Gamba P, Veening JW, Saunders NJ, Hamoen LW, Daniel RA: Two-step assembly dynamics of the bacillus subtilis divisome. J Bacterio/ 2009, 191(13):4186-4194.

18. Pichoff S, Lutkenhaus J: Overview of cell shape: cytoskeletons shape bacterial cells. Curr Opin Microbiol 2007, 10(6):601-605.

19. Graumann PL: Cytoskeletal elements in bacteria. Annu Rev Microbio/ 2007, 61:589-618

20. Jones $\amalg$, Carballido-Lopez R, Errington J: Control of cell shape in bacteria: helical, actin-like filaments in bacillus subtilis. Cell 2001, 104(6):913-922.

21. Lingwood D, Simons K: Lipid rafts as a membrane-organizing principle. Science 2010, 327(5961):46-50.

22. Browman DT, Hoegg MB, Robbins SM: The SPFH domain-containing proteins: more than lipid raft markers. Trends Cell Biol 2007, 17(8):394-402.

23. Langhorst MF, Reuter A, Stuermer CA: Scaffolding microdomains and beyond: the function of reggie/flotillin proteins. Cell Mol Life Sci 2005, 62(19-20):2228-2240.

24. Lopez D, Kolter R: Functional microdomains in bacterial membranes. Genes Dev 2010, 24(17):1893-1902.

25. Kaimer C, Gonzalez-Pastor JE, Graumann PL: SpollIE and a novel type of DNA translocase, $\mathrm{Sft} A$, couple chromosome segregation with cell division in bacillus subtilis. Mol Microbiol 2009, 74(4):810-825

26. Biller SJ, Burkholder WF: The bacillus subtilis SftA (YtpS) and SpollIE DNA translocases play distinct roles in growing cells to ensure faithful chromosome partitioning. Mol Microbiol 2009, 74(4):790-809.

27. Levin PA, Kurtser IG, Grossman AD: Identification and characterization of a negative regulator of FtsZ ring formation in bacillus subtilis. Proc Natl Acad Sci USA 1999, 96(17):9642-9647.

28. Harry EJ, Wake RG: The membrane-bound cell division protein DivIB is localized to the division site in bacillus subtilis. Mol Microbiol 1997, 25(2):275-283

29. Daniel RA, Noirot-Gros MF, Noirot $P$, Errington J: Multiple interactions between the transmembrane division proteins of bacillus subtilis and the role of FtsL instability in divisome assembly. J Bacteriol 2006, 188(21):7396-7404.

30. Hinderhofer M, Walker CA, Friemel A, Stuermer CA, Moller HM, Reuter A: Evolution of prokaryotic SPFH proteins. BMC Evol Biol 2009, 9:10

31. Donovan C, Bramkamp M: Characterization and subcellular localization of a bacterial flotillin homologue. Microbiology 2009, 155(Pt 6):1786-1799.

32. Glebov $O O$, Bright NA, Nichols BJ: Flotillin-1 defines a clathrinindependent endocytic pathway in mammalian cells. Nat Cell Biol 2006 8(1):46-54.

33. Wu $\sqcup$, Errington J: Coordination of cell division and chromosome segregation by a nucleoid occlusion protein in bacillus subtilis. Cell 2004 , 117(7):915-925.

34. Dempwolff F, Moller HM, Graumann PL: Synthetic motility and cell shape defects associated with deletions of flotillin/reggie paralogs in bacillus subtilis and interplay of these proteins with NfeD proteins. J Bacteriol 2012, 194(17):4652-4661.
35. Defeu Soufo HJ, Graumann PL: Actin-like proteins MreB and Mbl from bacillus subtilis are required for bipolar positioning of replication origins. Curr Biol 2003, 13(21):1916-1920.

36. Formstone A, Errington J: A magnesium-dependent mreB null mutant implications for the role of mreB in bacillus subtilis. Mol Microbiol 2005 55(6):1646-1657.

37. Lee $Y H$, Kingston AW, Helmann JD: Glutamate dehydrogenase affects resistance to cell wall antibiotics in bacillus subtilis. J Bacterio/ 2011, 194(5):993-1001

38. Jaacks KJ, Healy J, Losick R, Grossman AD: Identification and characterization of genes controlled by the sporulation regulatory gene spoOH in bacillus subtilis. J Bacteriol 1989, 171:4121-4129.

39. Feucht A, Lewis PJ: Improved plasmid vectors for the production of multiple fluorescent protein fusions in Bacillus subtilis. Gene 2001, 264(2):289-297.

40. Defeu Soufo HJ, Graumann PL: Dynamic localization and interaction with other bacillus subtilis actin-like proteins are important for the function of MreB. Mol Microbiol 2006, 62:1340-1356.

41. Gueiros-Filho FJ, Losick R: A widely conserved bacterial cell division protein that promotes assembly of the tubulin-like protein FtsZ. Genes Dev 2002, 16(19):2544-2556.

42. Dempwolff F, Reimold C, Reth M, Graumann PL: Bacillus subtilis MreB orthologs self-organize into filamentous structures underneath the cell membrane in a heterologous cell system. PLoS One 2011, 6(11):e27035.

43. Kidane D, Sanchez H, Alonso JC, Graumann PL: Visualization of DNA double-strand break repair in live bacteria reveals dynamic recruitment of bacillus subtilis $\mathrm{RecF}, \mathrm{RecO}$ and $\mathrm{RecN}$ proteins to distinct sites on the nucleoids. Mol Microbiol 2004, 52(6):1627-1639.

44. Defeu Soufo HJ, Reimold C, Linne U, Knust T, Gescher J, Graumann PL: Bacterial translation elongation factor EF-Tu interacts and colocalizes with actin-like MreB protein. Proc Natl Acad Sci USA 2010, 107(7):3163-3168.

45. Waidner B, Specht M, Dempwolff F, Haeberer K, Schaetzle S, Speth V, Kist M, Graumann PL: A novel system of cytoskeletal elements in the human pathogen helicobacter pylori. PLoS Pathog 2009, 5(11):e1000669.

doi:10.1186/1471-2180-12-298

Cite this article as: Dempwolff et al:: The deletion of bacterial dynamin and flotillin genes results in pleiotrophic effects on cell division, cell growth and in cell shape maintenance. BMC Microbiology 2012 12:298.

\section{Submit your next manuscript to BioMed Central and take full advantage of:}

- Convenient online submission

- Thorough peer review

- No space constraints or color figure charges

- Immediate publication on acceptance

- Inclusion in PubMed, CAS, Scopus and Google Scholar

- Research which is freely available for redistribution 